\title{
Use of oral contraceptives in $B R C A$ mutation carriers and risk for ovarian and breast cancer: a systematic review
}

\author{
D. Huber ${ }^{1} \cdot$ S. Seitz ${ }^{1} \cdot$ K. Kast ${ }^{2} \cdot$ G. Emons ${ }^{3} \cdot$ O. Ortmann ${ }^{1}$ (I)
}

Received: 25 October 2019 / Accepted: 27 January 2020 / Published online: 5 March 2020

(C) The Author(s) 2020, corrected publication 2021

\begin{abstract}
Purpose BRCA mutation carriers have an increased risk of developing breast or ovarian cancer. Oral contraception (OC) is known to increase breast cancer and reduce ovarian cancer risk in the general population. This review analyses the published data on OC and risk of cancer in BRCA mutation carriers.

Methods We included all relevant articles published in English from 1995 to 2018. Literature was identified through a search on PubMed and Cochrane Library.

Results We included four meta-analyses, one review, one case-control study and one retrospective cohort study on the association between ovarian cancer and OC in BRCA mutation carriers. All report a risk reduction for the OC users and several also describe an inverse correlation with duration of use. Regarding breast cancer, we included four meta-analyses, one review, one case-control study, two case-only studies, one prospective and one retrospective cohort study. Some studies report a risk elevation, while others did not find an association between OC use and breast cancer in BRCA mutation carriers. In other studies, the association was limited to early-onset breast cancer and/or associated with young age at first start of OC. Conclusion Oral contraception leads to a risk reduction of ovarian cancer also in BRCA mutation carriers. An increase in breast cancer risk due to OC cannot be excluded. Women with BRCA mutation who consider OC use have to be informed about possible increase in breast cancer risk and alternative contraceptive methods. OC should not be used for the prevention of ovarian cancer in this population.
\end{abstract}

Keywords Oral contraception $\cdot$ Breast cancer $\cdot$ Ovarian cancer $\cdot B R C A 1 \cdot B R C A 2$

\section{Introduction}

Mutations in BRCA1/2 genes represent significant risk factors for breast and ovarian cancer. A recent prospective study suggests a cumulative risk to the age of 80 years in BRCA1 mutation carriers of up to $72 \%$ for breast cancer and up to

D. Huber and S. Seitz contributed equally to this work.

O. Ortmann

olaf.ortmann@klinik.uni-regensburg.de

1 Department of Gynecology and Obstetrics, University Medical Center Regensburg, Regensburg, Germany

2 Department of Gynecology and Obstetrics, Medical Faculty and University Hospital Carl Gustav Carus, TU Dresden, Dresden, Germany

3 Department of Gynecology and Obstetrics, Georg August University Göttingen, University Medicine, Göttingen, Germany
$44 \%$ for ovarian cancer. In $B R C A 2$ mutation carriers, the cumulative breast cancer risk to the age of 80 years according to this data is increased up to $69 \%$ and for ovarian cancer up to $17 \%$, respectively [1].

The estimated prevalence of mutations in BRCAl and 2 genes varies between 0.3 and $0.8 \%$. Depending on the investigated population, the prevalence of founder mutations can be significantly higher [2]. Besides the genetic risk factors, there are other risk modifying factors, e.g. endocrine interventions. Among these, one of the most common is oral contraception (OC). In the general population, current or recent use of oral contraceptives leads to an increased risk for breast cancer $(\mathrm{RR}=1.20$; 95\% CI 1.14-1.2). The risk caused by combined oral contraceptives appears to vary depending on the duration of use and the type of progestin [3]. In contrast, use of OC leads to a profound decrease of ovarian cancer risk by $20 \%$ RR reduction for every 5 years of use. This effect persists for many years after cessation of use 
[4]. There is little data on the combined effects of various risk factors, such as obesity, exercise or genetic mutations. In this review, we analyse the published data on $\mathrm{OC}$ and risk for breast or ovarian cancer in $B R C A 1 / 2$ mutation carriers.

\section{Methods}

A search for relevant articles was run from 1995 to 2018 on PubMed. The MeSH used for the search were "oral contraceptives" and "BRCA mutation" and "oral contraceptives" and "breast cancer" or "ovarian cancer". The search created 59, 1634 and 1146 hits, respectively. We also conducted a search within the Cochrane library. There were no Cochrane reviews available on OC and risk of cancer in BRCA1/2 mutation carriers. The hits were searched for relevance, clinical trials, reviews and meta-analyses. Also, the references of appropriate articles were screened for relevant publications. The authors of this review defined all those articles which deal with the specific subject of whether or not OC has an impact on breast and/or ovarian cancer in BRCAl/2 mutation carriers as relevant.

We found 31 relevant publications, 7 of which were regarding both cancer risks, 8 regarding the risk of ovarian cancer and 16 regarding breast cancer risk only. After excluding cross-match, i.e. excluding publications which were part of an included review or meta-analysis, there remained a total of 13 publications to include in our review. Among those, there were four meta-analyses and one systematic review regarding both ovarian and breast cancer risk. Furthermore, we included one case-control study and one retrospective cohort study on ovarian cancer risk and one case-control, two case-only studies, one prospective and one retrospective cohort study on OC-induced breast cancer risk in $B R C A$ mutation carriers. Due to differences in populations, study types, statistical methodology and inclusion criteria, the studies included in this review were not appropriate for meta-analysis.

\section{Results}

\section{Ovarian cancer}

After excluding cross-match, we included seven publications on ovarian cancer risk associated with OC use in $B R C A$ mutation carriers. Among those, there were four meta-analyses, one review, one case-control study and one retrospective cohort study. All of them report a risk reduction for the OC users and several also describe an inverse correlation with ovarian cancer risk and duration of OC use (Table 1).

A case-control study from 1998 by Narod et al. [5] found a risk reduction associated with ever use compared to never use of OC. The risk reduction was present in $B R C A 1$ $(\mathrm{OR}=0.5 ; 95 \%$ CI $0.3-0.9)$ and $B R C A 2(\mathrm{OR}=0.4 ; 95 \% \mathrm{CI}$ 0.2-1.1) mutation carriers, with a limited number of BRCA2 mutation carriers included in the study. There was an inverse correlation with ovarian cancer risk and duration of OC ( $p$ for trend $<0.001$ ), with a risk reduction of $60 \%$ for a duration of OC use for 6 years and more. The average duration of OC was 4 years for cases and 6 years for control women. The mean age of beginning OC use was 24 years for cases and 22 years for controls. The controls were living sisters of the patients and they were included whether or not information on molecular testing was available. Also, $30 \%$ of the patients and $18 \%$ of the controls had a history of breast cancer and more than one-third of the controls had undergone bilateral oophorectomy before enrollment. Specific ethnic groups were slightly overrepresented, that is Ashkenazi Jewish within the cases and French-Canadian women within the control group.

A study by Iodice et al. [6] from 2010 included four case-control studies and one retrospective cohort study. The study included 1262 cases and 2678 controls with BRCAI mutation, 253 cases and 538 controls with $B R C A 2$ mutation and 1 case with $B R C A 1$ and 2 mutation. A meta-analysis confirmed significantly reduced risk for ovarian cancer for BRCA1 (SRR $=0.51 ; 95 \%$ CI 0.40-0.65) and BRCA2 mutations carriers ( $\mathrm{SRR}=0.50 ; 95 \%$ CI $0.29-0.89)$ associated with use of OC. Also, increasing duration of OC use was associated with a linear decrease in risk of $36 \%$ for each additional 10 years (95\% CI 22-47\%; $p<0.01)$. The definition for use of OC was $>1$ year in one of the included case-control study [7] and any duration of use in the rest of the studies. The mean age at enrollment varied between 41 [8] and 53 years $[9,10]$. Analysis of age at the beginning of use and its association with ovarian cancer risk was not performed. All of the included studies were retrospective and their designs partly differed.

A review by Cibula et al. [11] included five case-control studies and one retrospective cohort study. The included studies comprise a total of 1203 cases and 2160 controls with BRCA1 mutation, 277 cases and 432 controls with BRCA2 mutation, 1 case with BRCAl and 2 mutation and 282 cases with not further indicated BRCA1/2 mutation carriers. Three of the included studies $[7,8,10]$ were included in the meta-analysis by Iodice [6]. Of the five studies included in the review, only one did not confirm a protective effect for ovarian cancer in BRCA mutation carriers (OR for 5 or more years of OC use $=1.07 ; 95 \%$ CI $0.63-1.83$ ) [12]. The review provides the specific ethnic/Jewish background and the small number of OC users as possible explanations. The five other studies showed a decrease of ovarian cancer risk associated with OC use. In some of the studies, the protective effect was associated with duration of OC use of at least 1 year [7, 12]. One of the included studies found a protective 
Table 1 Oral contraception and risk of ovarian cancer in BRCA mutation carriers

\begin{tabular}{|c|c|c|}
\hline $\begin{array}{l}\text { Study/study design/Oxford Center of } \\
\text { Evidence-based Medicine (OCEBM) level of } \\
\text { evidence (LOE) }\end{array}$ & Number & Results \\
\hline $\begin{array}{l}\text { Narod et al. [5] } \\
\text { Case-control study } \\
\text { LOE } 3 b\end{array}$ & $\begin{array}{l}\text { Cases: } 179 \text { BRCA1, } 28 \text { BRCA2 } \\
\text { Controls: } 161 \text { (50 BRCA1, } 3 \text { BRCA2, } 42 \text { with- } \\
\text { out mutation, } 66 \text { without testing) }\end{array}$ & $\begin{array}{l}\text { Risk reduction } \\
\text { BRCA1: } \mathrm{OR}=0.5 ; 95 \% \text { CI } 0.3-0.9 \\
\text { BRCA2: } \mathrm{OR}=0.4 ; 95 \% \text { CI } 0.2-1.1 \\
\text { Combined: } \mathrm{OR}=0.5 ; 95 \% \text { CI } 0.3-0.8 \\
\text { Inverse correlation with duration of } \mathrm{OC}(p \\
\text { trend }<0.001)\end{array}$ \\
\hline $\begin{array}{l}\text { Iodice et al. [6] } \\
\text { Meta-analysis } \\
\text { LOE 2a } \\
\text { Included studies } \\
\text { Four case-control studies }[7,9,10,14] \\
\text { One retrospective cohort study }[8]\end{array}$ & $\begin{array}{l}\text { Cases: } 1262 \text { BRCA1, } 253 \text { BRCA2, } 1 \text { BRCA1+2 } \\
\text { Controls: } 2678 \text { BRCA1, } 538 \text { BRCA2 }\end{array}$ & $\begin{array}{l}\text { Risk reduction } \\
B R C A 1: \mathrm{SRR}=0.51 ; 95 \% \text { CI } 0.40-0.6 \\
B R C A 2: \mathrm{SRR}=0.50 ; 95 \% \text { CI } 0.29-0.89 \\
\quad(p=0.88) \\
\text { Combined: } \mathrm{SRR}=0.50 ; 95 \% \text { CI } 0.33-0.75 \\
\text { Linear decrease in risk of } 36 \% / 10 \text { years }(95 \% \\
\text { CI } 22-47 \%, p \text { trend }<0.01)\end{array}$ \\
\hline $\begin{array}{l}\text { Cibula et al. [11] } \\
\text { Review } \\
\text { LOE } 2 \mathrm{a}\end{array}$ & $\begin{array}{l}\text { Cases: } 1203 \text { BRCA1, } 277 \text { BRCA2, } 1 \\
\text { BRCA1+2, 282 BRCA1/2 (not further indi- } \\
\text { cated, from [25]) }\end{array}$ & $\begin{array}{l}\text { Risk reduction in all but one [12] of the } \\
\text { included studies } \\
\text { Partly inverse correlation with duration of OC }\end{array}$ \\
\hline
\end{tabular}

\section{Included studies}

Five case-control studies [7, 10, 12, 25, 38]

One retrospective cohort study [8]

Controls: 2160 BRCA1, 432 BRCA2

Cibula et al. [13]

Meta-analysis

LOE $2 \mathrm{a}$

Included studies

Three case-control studies [7, 10, 14]
Cases: 934 BRCA1, 161 BRCA2, 1 BRCA1+2

Controls: 2307 BRCA1, 413 BRCA2
Risk reduction

BRCA1: OR $=0.56 ; 95 \%$ CI $0.49-0.69$ $(p<0.001)$

BRCA2: $\mathrm{OR}=0.49,95 \%$ CI $0.32-0.77$ $(p<0.002)$

Combined: $\mathrm{OR}=0.57 ; 95 \%$ CI $0.47-0.70$ $(p<0.001)$

Inverse correlation with duration of $\mathrm{OC}$ : $\mathrm{OR}=0.95$; 95\% CI 0.93-0.97 $(p<0.001)$

\author{
Moorman et al. [15] \\ Meta-analysis \\ LOE $2 \mathrm{a}$ \\ Included studies \\ Five case-control studies [7, 10, 12, 14, 38] \\ One retrospective cohort study [8]
}

\section{Friebel et al. [16]}

Review and meta-analysis

LOE 2a

Included studies

Four case-control studies [10, 14, 17, 18]

One retrospective cohort study [8]

Perri et al. [19]

Retrospective cohort study

LOE $2 b$
Cases: 1353 BRCA1, 277 BRCA2

Controls: 2310 BRCA1, 423 BRCA2

Risk reduction

BRCA1: OR $=0.55 ; 95 \%$ CI $0.47-0.66$ $(p=0.743)$

BRCA2: $\mathrm{OR}=0.65 ; 95 \%$ CI $0.34-1.24$ $(p=0.096)$ $(p=0.210)$

Cases: 1348 BRCA1, 239 BRCA2, 1 BRCA1+2 Risk reduction

Controls: 2926 BRCA1, 439 BRCA2

$B R C A 1$ : Risk reduction associated with use. Risk reduction of $33-80 \%$ for OC use $>1$ year

$B R C A 2$ : Risk reduction of $58-63 \%$ associated with use

Cases: 139 BRCA1, 33 BRCA2, 3 unknown

Controls: 579 BRCA1, 298 BRCA2, 3

$B R C A 1+2,19$ unknown
Combined: $\mathrm{OR}=0.58 ; 95 \%$ CI $0.46-0.73$
Risk reduction

BRCA1: OR $=0.21 ; 95 \%$ CI $0.14-0.33$

$(p<0.001)$

BRCA2: $\mathrm{OR}=0.24 ; 95 \%$ CI $0.09-0.61$ $(p<0.001)$

Combined: $\mathrm{OR}=0.19 ; 95 \%$ CI $0.13-0.28$ $(p<0.001)$

Inverse correlation with duration of $\mathrm{OC}$ effect associated with use of $\mathrm{OC}$, restricted to $B R C A 1$ mutation carriers $(\mathrm{HR}=0.52 ; 95 \% \mathrm{CI} 0.37-0.73 ; p=0.0002)$ [8]. Some of the studies included only BRCAl mutation carriers or a restricted number of $B R C A 2$ mutation carriers. The largest study included 1 ovarian cancer case with $B R C A 1$ and 2 mutation, 670 ovarian cancer cases and 2043 controls with BRCA1 mutation as well as 128 cases and 380 controls with BRCA2 mutation [10]. It confirmed a risk reduction for both BRCA1 $(\mathrm{OR}=0.56 ; 95 \%$ CI $0.45-0.71, p<0.0001)$ and $B R C A 2$ mutation carriers $(\mathrm{OR}=0.39 ; 95 \%$ CI $0.23-0.66$, $p=0.0004$ ) associated with use of OC. Also, a significant trend of risk reduction associated with increasing duration 
of use was reported $(p<0.0001)$ in this study. Not all of the studies included in the review provided molecular testing of all controls. All of the studies were retrospective and the designs were different.

A meta-analysis by the same author confirms a protective effect for any past use of OC and a trend in risk with increasing duration of OC use [13]. It includes three case-control studies, one of which [14] included only BRCAl mutation carriers. In the meta-analysis, the protective effect was shown for BRCA1 $(\mathrm{OR}=0.56 ; 95 \%$ CI $0.49-0.69 ; p<0.001)$ as well as BRCA2 mutation carriers $(\mathrm{OR}=0.49,95 \% \mathrm{CI}$ $0.32-0.77 ; p<0.002)$. The OR for pooled trend in risk with increasing duration of OC use was 0.95 (95\% CI 0.93-0.97; $p<0.001)$. The age at start of use and its association with ovarian cancer risk was not studied, neither were type and dosage of OC.

The most recent meta-analysis by Moorman et al. is also the largest of the included studies, considering 1353 ovarian cancer cases and 2310 controls with $B R C A 1$ mutation as well as 277 cases and 423 controls with $B R C A 2$ mutation [15]. Comparing ever use of and never use of OC, the OR for BRCA1 mutation carriers was 0.55 (95\% CI $0.47-0.66$ ). The OR for BRCA2 mutation carriers was 0.65 (95\% CI 0.34-1.24), the difference between the two groups was not statistically significant $(p=0.975)$. All of the included studies demonstrated an inverse association between ovarian cancer risk and duration of $\mathrm{OC}$ use. However, these findings could not be used for the meta-analysis due to differences in duration categories. Only one of the studies reported an effect by time since last OC use, with a lower risk for more recent users ( $<10$ years) [8]. As none of the studies listed type and dose of contraceptive pills, the possible effect of different combinations and dosage of OC could not be evaluated. Mean age at OC start and duration was not reported. All of the included studies were observational and some did not exclude prevalent cancer cases.

Friebel et al. [16] conducted a systematic review and meta-analysis on modifiers of cancer risk in BRCA 1 and 2 carriers. Regarding OC in BRCA mutation carriers, they included four case-control studies and one retrospective cohort study with a total of 1348 cases and 2926 controls with $B R C A 1$ mutation, 239 cases and 439 controls with $B R C A 2$ mutation and 1 case with $B R C A 1+2$ mutation. For $B R C A 1$ mutation carriers, four of the included studies reported a risk reduction of ovarian cancer associated with use of OC $[8,10,14,17]$. One of the studies showed no association between OC and ovarian cancer risk [18]. Regarding the duration of OC use, all of the included studies that examined $\mathrm{OC}$ use $>1$ year showed a statistically significant risk reduction from 33 to $80 \%$ in BRCAl mutation carriers $[8,10,14]$. Due to overlapping samples, no meta-analysis could be performed. For BRCA2 mutation carriers, two of the included studies reported a risk reduction from 58 to
$63 \%$ associated with use of OC $[10,17]$. Data were not sufficient to perform a meta-analysis in BRCA2 mutation carriers and the duration of use was not further examined. Age at start of use or the type of OC were not reported.

Perri et al. [19] conducted a study on ovarian cancer risk for $B R C A$ mutation carriers undergoing fertility treatment. All of the participants were Jewish Israeli women with personal or family history of BRCA mutation-associated cancers. A multivariate analysis showed a reduced risk for BRCAl (OR $=0.21 ; 95 \%$ CI 0.14-0.33) and BRCA2 mutation carriers $(\mathrm{OR}=0.21 ; 95 \%$ CI $0.09-0.61)$ associated with use of OC. There was a further risk reduction with duration of use: the OR for up to 1 year of OC use was 0.36 (95\% CI $0.16-0.84)$, for more than 5 years 0.10 (95\% CI 0.06-0.17). Women who had undergone risk-reducing oophorectomy were not excluded from the study. The mean age was 53.6 years for cases and 49.1 for controls. There was no information on age at start of use, duration or type of OC.

\section{Breast cancer}

The included studies on the association between OC use and breast cancer risk in BRCA mutation carriers comprise four meta-analyses, one review, one case-control-study, two case-only studies, one retrospective and one prospective cohort study. Some of the studies report a risk elevation, while others did not find an interaction between OC use and breast cancer (Table 2).

Pasanisi et al. [20] conducted a case-only study which resulted in a borderline significant association between genetic breast cancer and OC use compared with never use $(\mathrm{OR}=1.3 ; 95 \%$ CI 1.0-1.7). The highest association was found for OC start between 18 and 20 years (OR, 1.6; 95\% CI 1.1-2.3). From the results, the research group inferred a higher vulnerability to OC for women with $B R C A$ mutation. Women with breast cancer before the age of 45 years who were then classified as sporadic or genetic cases were included. There was no systematic genetic testing and classification of genetic cases was based on software-assisted mathematical $B R C A$ mutation probability. The duration of OC use was analysed as well. However, the comparison between duration of more than 5 years and a shorter duration of maximum 5 years of use was not statistically significant. There was no information on type and dosage of OC.

In the meta-analysis by Iodice et al. [6], not only the impact of $\mathrm{OC}$ on breast cancer, but also on ovarian cancer risk in $B R C A$ mutation carriers was examined. A minority of the included women had a history of ovarian cancer [21]. The meta-analysis included four case-control studies and one retrospective cohort study. The study found no significant association between $\mathrm{OC}$ and breast cancer risk for $B R C A 1$ (RR $=1.09$; 95\% CI 0.77-1.54) and BRCA2 mutation carriers $(\mathrm{RR}=1.15 ; 95 \% \mathrm{CI} 0.61-2.18)$. An association 
Table 2 Oral contraception and risk of breast cancer in BRCA mutation carriers

\begin{tabular}{lll}
\hline Study/study design/Oxford Center of & Number & Results \\
Evidence-based Medicine (OCEBM) level of & &
\end{tabular}
evidence (LOE)

Pasanisi et al. [20]

Case-only study

382 "genetic", 1333 "sporadic" cases

LOE 4

Iodice et al. [6]

Meta-analysis

LOE 2a

Included studies

Four case-control studies [14, 21, 25, 28]

One retrospective cohort study [26]

Cibula et al. [11]

Review

LOE $2 \mathrm{a}$

Included studies

Seven case-control studies [21, 25, 27, 28, 39-41]

One retrospective cohort study [26]

Cibula et al. [13]

Meta-analysis

LOE 2a

Included studies

Three case-control studies [14, 25, 28]

Two retrospective cohort studies [21, 26]

Five case-case studies [23, 24, 27, 40, 42]

Moorman et al. [15] Meta-analysis

LOE 2a

Included studies

Three case-control studies [14, 25, 28]

Two retrospective cohort studies [26, 29]

Kotsopoulos et al. [30]

Case-control study

LOE $3 b$

Friebel et al. [16]

Review and meta-analysis

LOE 2a

Included studies:

Five case-control studies: [14, 21, 25, 28, 32]

Two retrospective cohort studies [26, 29]

Rieder et al. [33]

Case-only study

Cases: 2154 BRCA1, 707 BRCA2

Controls: 2280 BRCA1, 672 BRCA2

Cases: 2151 BRCA1, 862 BRCA2, 94

$B R C A 1 / 2$ (not further indicated, from [40])

Controls: 2121 BRCA1, 719 BRCA2

Case-control studies:

Cases: 1524 BRCA1, 458 BRCA2

Controls: 1631 BRCA1, 509 BRCA2

Cohort studies:

Cases: 630 BRCA1, 249 BRCA2

Controls: 649 BRCA1, 163 BRCA2

Case-case studies:

131 BRCA1, 80 BRCA1, 1 BRCA1+2, 94

$B R C A 1 / 2$ (not further indicated, from [40])

Cases:

2401 BRCA1, 830 BRCA2

Controls:

2215 BRCA1, 672 BRCA2, 373 BRCA1/2 (not

further indicated, from [29])

2492 BRCA1 case-control pairs

Case-control studies:

Cases: 3606 BRCA1, 1257 BRCA2

Controls: 3730 BRCA1, 1308 BRCA2

Cohort studies:

Cases: 877 BRCA1, 372 BRCA2

Controls: 584 BRCA1, 163 BRCA2, 373

$B R C A 1 / 2$ (not further indicated, from [29])

258 BRCA1, 108 BRCA2
Borderline significant association

Genetic cases: $\mathrm{OR}=1.3 ; 95 \%$ CI $1.0-1.7$ $(p=0.05)$

Highest association for OC start at 18-20 years: $\mathrm{OR}=1.6 ; 95 \%$ CI $1.1-2.3(p$ trend $=0.18)$

Duration of use not statistically significant $(p=0.32)$

No significant association

BRCA1: RR $=1.09 ; 95 \%$ CI $0.77-1.54$

BRCA2: $\mathrm{RR}=1.15 ; 95 \%$ CI $0.61-2.18$

Combined: $\mathrm{SRR}=1.33 ; 95 \%$ CI $0.88-1.45$ )

No association with duration of use $(p=0,2)$

Mild to moderate increase in risk

Further increase in risk when OC duration $\geq 4$ years before FFT (BRCA1:

$\mathrm{HR}=1.49$; 95\% CI 1.05-2.11. BRCA 2:

$\mathrm{HR}=2.58 ; 95 \%$ CI $1.21-5.49$ )

No significant association

BRCA1: $\mathrm{OR}=1.08 ; 95 \%$ CI $0.94-1.25$

$(p=0.25)$

BRCA2: $\mathrm{OR}=1.03 ; 95 \%$ CI $0.81-1.32$ $(p=0.788)$

Increase in risk

BRCA1: $\mathrm{OR}=1.48 ; 95 \%$ CI $1.14-1.92$ $(p=0.727)$

No increase in risk

BRCA1/2: OR $=0.80 ; 95 \%$ CI $0.59-1.08$ $(p=0.147)$

Non-statistically relevant increase in risk

BRCA1: OR $=1.19 ; 95 \%$ CI 0.92-1.55 $(p=0.004)$

BRCA2: $\mathrm{OR}=1.36 ; 95 \%$ CI $0.89-2.10$ $(p=0.022)$

Combined: OR $=1.21 ; 95 \%$ CI $0.93-1.58$ $(\mathrm{p}<0.001)$

Increase in risk when starting $<20$ years: $\mathrm{OR}=1.45 ; 95 \%$ CI $1.20-1.75(p=0.0001)$

No statistically relevant increase in risk when starting at $20-25$ years: $\mathrm{OR}=1.19 ; 95 \% \mathrm{CI}$ $0.99-1.42(p=0.06)$

Effect only for early-onset $<40$ years: $\mathrm{OR}=1.40 ; 95 \%$ CI $1.14-1.70(p=0.001)$

No association

BRCA1: $\mathrm{ES}=0.78 ; 95 \%$ CI $0.59-1.04$

BRCA2: $\mathrm{ES}=1.04 ; 95 \%$ CI $0.81-1.32[25,28]$

Increase in risk

BRCA1: $\mathrm{ES}=1.59 ; 95 \%$ CI 1.32-1.92

BRCA2: $\mathrm{ES}=1.85 ; 95 \%$ CI $1.30-2.64$

No association with duration of use

Prior or current OC associated with younger age at diagnosis: $\mathrm{HR}=1.7 ; 95 \% \mathrm{CI} 1.1-2.05$ $(p=0.006)$

No association with duration of use: $\mathrm{HR}=1.00$; 95\% CI 0.99-1.00 
Table 2 (continued)

Study/study design/Oxford Center of

Number

Results

Evidence-based Medicine (OCEBM) level of evidence (LOE)

Park et al. [34]

Retrospective cohort study

LOE $2 b$

Schrijver et al. [35]

Retrospective and prospective cohort study LOE $1 b$
Cases: 168 BRCA1, 109 BRCA2
Controls: 54 BRCA1, 250 BRCA2

Prospective cohort:

Cases: 269 BRCA1, 157 BRCA2

Controls: 2007 BRCA1, 1453 BRCA2

Retrospective cohort, left-truncated:

Cases: 1095 BRCA1, 752 BRCA2

Controls: 2733 BRCA1, 1760 BRCA2

Retrospective full-cohort:

Cases: 2525 BRCA1, 1548 BRCA2

Controls: 3180 BRCA1, 1973 BRCA2

\author{
No significant association \\ BRCAl: $\mathrm{HR}=1.24 ; 95 \%$ CI $0.45-3.40$ \\ BRCA2: $\mathrm{HR}=0.71 ; 95 \%$ CI $0.21-2.37$ \\ No association for BRCAl (HR $=1.08 ; 95 \%$ \\ CI 0.75-1.5), increase in risk for $B R C A 2$ \\ $(\mathrm{HR}=1.75$; 95\% CI 1.03-2.9) \\ Increase in risk for $B R C A 1(\mathrm{HR}=1.26 ; 95 \% \mathrm{CI}$ \\ 1.06-1.51), no association for BRCA2 (1.06; \\ 95\% CI 0.85-1.33) \\ Increase in risk for $B R C A 1(\mathrm{HR}=1.39 ; 95 \% \mathrm{CI}$ \\ $1.23-1.58)$ \\ and $B R C A 2(\mathrm{HR}=1.52 ; 95 \%$ CI $1.28-1.81)$ \\ Inverse correlation with duration of use, \\ especially before FFTP (BRCA1: both retro- \\ spective analyses, $p<0.001$ and $p=0.001$; \\ $B R C A 2$ : full retrospective analysis, $p=0.002$ )
}

between duration of use was not found, either. A significant increased risk was found for OC formulations before 1975 (RR 1.47; 95\% CI 1.06-2.04), but not for more recent preparations (RR: $1.17 ; 95 \%$ CI 0.74-1.86). The mean age of women varied between 33 [22] and 45 years [23]. The definition of OC use was more than 1 year in one [24] and any duration of use in the rest of the included studies. All of them were retrospective and the study designs were not identical. Some included controls even if molecular testing was not available. Genetic testing of the enrolled controls in the metanalysis was therefore not complete.

The large review conducted by Cibula et al. [11] from 2010 included a total of seven case-control and one retrospective cohort study. The studies mostly found a mild or moderate risk elevation, but the power was low. The largest study included 981 case-control pairs with BRCA1 and 330 pairs with $B R C A 2$ mutations [25]. An elevated risk associated with OC use was found only for BRCA1 mutation carriers with an early diagnosis before the age of 40 years $(\mathrm{OR}=1.38 ; 95 \% \mathrm{CI} 1.11-1.72)$. An elevated risk was not detected for $B R C A 1 / 2$ mutation carriers who developed breast cancer after the age of 40 years. The second largest study included a cohort of $1181 \mathrm{BRCAl}$ and 412 BRCA2 mutation carriers [26]. Of those, 597 BRCA1 and $249 B R C A 2$ mutation carriers were diagnosed with breast cancer. An elevated risk associated with OC use was found for both $B R C A 1$ (HR $=1.47 ; 95 \% \mathrm{CI} 1.13-1.91$ ) and $B R C A 2$ mutation carriers $(\mathrm{HR}=1.49 ; 95 \%$ CI 0.82-2.70). A further increase in risk was seen when the duration of OC use was at least 4 years before the first full-term pregnancy (FFTP); the observed HRs were not significantly different from those in women without family history $(\mathrm{BRCA} 1: \mathrm{HR}=1.49 ; 95 \%$ CI 1.05-2.11. BRCA 2:
$\mathrm{HR}=2.58 ; 95 \%$ CI 1.21-5.49) [26]. The included studies were all retrospective. Study designs were not identical and some of the studies matched cases with controls without mutation $[27,28]$. The authors conclude that OC use might be associated with a weak risk elevation for breast cancer in patients with $B R C A$ mutation, but the risk-benefit balance is influenced positively by the protective effect on ovarian cancer.

In their meta-analysis from 2011, Cibula et al. [13] also examined the association of $\mathrm{OC}$ with breast cancer in $B R C A$ mutation carriers. Three case-control, two retrospective cohort and five case-case studies were included. 27 of the BRCA 1 mutation carriers, either cases or controls, had a history of ovarian cancer [21]. A meta-analysis was carried out separately for the different study types. In case-control studies, inverse variance pooling did not find a relevant risk elevation associated with $\mathrm{OC}$ for $B R C A I(\mathrm{OR}=1.08 ; 95 \% \mathrm{CI}$ 0.94-1.25) and $B R C A 2$ (OR $=1.03$; 95\% CI 0.811 .32$)$. Likewise, no elevation of risk was found for $B R C A 1 / 2$ mutation carriers in case-case studies ( $\mathrm{OR}=0.80 ; 95 \%$ CI 0.59-1.08). However, a meta-analysis of the cohort studies showed a significant increase in risk associated with OC use for BRCA1 mutation carriers ( $\mathrm{OR}=1.48 ; 95 \%$ CI 1.14-1.92). This result was mainly driven by one study which represented 98\% weight of the data sample set [26]. Exposure to OC was defined as use for more than 1 year in one case-control and one case-case study, use for more than 3 months in one cohort study and as long-term use in one case-case study $[21,24,27,28]$. In all of the other studies, OC exposure was defined as use. A trend in risk with duration of use could not be evaluated due to different populations and study designs. Information on type and dosage of OC was 
not given. Overall, the included studies showed inconsistent results and many of them had a limited group size.

In their meta-analysis, Moorman et al. [15] included three case-control studies and two retrospective cohort studies. The case-control $[14,25,28]$ and one of the cohort studies [26] are identical with those in the meta-analysis by Cibula et al. in 2011 [13]. The authors found a risk elevation associated with $\mathrm{OC}$ use for $B R C A l(\mathrm{OR}=1.19 ; 95 \% \mathrm{CI} 0.92-1.55)$ and $B R C A 2$ mutation carriers (OR 1.36; 95\% CI 0.89-2.10) which was not statistically significant. They draw the conclusion that the association between breast cancer and use of OC among BRCA mutation carriers does not differ greatly from the general population. The duration and timing of use could not be further examined due to inadequate data. A possible effect of type and dosage of OC could not be examined, either. The included studies were all observational and study designs were not identical. Some of the studies did not exclude prevalent cancer cases and some included patients from specific ethnic subgroups, such as Ashkenazi Jewish and Polish women [14, 29].

Kotsopoulos et al. included 2492 case-control pairs with BRCAl mutation. They found an increase in breast cancer risk for BRCAl mutation carriers who started OC before the age of 20 years $(\mathrm{OR}=1.45$; 95\% CI $1.20-1.75$; $p=0.0001)$ and a non-significant increase for carriers who started between 20 and 25 years of age (OR 1.19; 95\% CI $0.99-1.42 ; p=0.06$ ) [30]. When adjusted for age at diagnosis, the effect was observed only for early-onset breast cancer with diagnosis before the age of 40 years $(\mathrm{OR}=1.40 ; 95 \%$ CI 1.14-1.70; $p=0.001)$. When breast cancer was diagnosed at or after 40 years of age, no increase in risk was reported (OR 0.97; 95\% CI 0.79-1.20; $p=0.81$ ). The association between use of $\mathrm{OC}$ and early-onset breast cancer was strongest for women who started OC before the age of 20 years $(\mathrm{OR}=1.74 ; 95 \%$ CI 1.36-2.22; $p=0.00001)$. An increase in risk of early-onset breast cancer was also observed when the age at $\mathrm{OC}$ start was between 20 and 25 years $(\mathrm{OR}=1.36$, $1.07-1.73 ; p=0.02)$. The mean age at diagnosis in the study was 39.7 years, the mean age at recruitment 46.3 years. The mean duration of use was 3.8 for cases and 3.5 years for controls. The association between breast cancer risk and time since last $\mathrm{OC}$ use was also examined. Compared with never use, the study found no association between current $\mathrm{OC}$ use and breast cancer risk ( $\mathrm{OR}=0.8 ; 95 \%$ CI $0.66-0.97) .5$ or more years after stopping OC use, a significant increase in risk of $38 \%$ was observed $(\mathrm{OR}=1.38 ; 95 \%$ CI $1.18-1.61)$. Information on type and dosage of $\mathrm{OC}$ was not provided.

Friebel et al. [16] included five case-control and two retrospective cohort studies in their review and meta-analysis regarding $\mathrm{OC}$ use and breast cancer risk in $B R C A$ mutation carriers. Two of the studies included only BRCAl mutation carriers [21, 31]. In total, the included studies comprised 4483 cases and 4314 controls with BRCAl mutation, 1629 cases and 1471 controls with $B R C A 2$ mutation as well as 373 controls with no further indicated $B R C A 1 / 2$ mutation. For $B R C A 1$ mutation carriers, the meta-analysis of the case-control studies did not find an association between use of OC and breast cancer risk ( $\mathrm{ES}=0.78 ; 95 \% \mathrm{CI} 0.59-1.04)$. The meta-analysis of the cohort studies, however, showed an increase in breast cancer risk associated with use of OC in BRCA1 mutation carriers ( $\mathrm{ES}=1.59$; 95\% CI 1.32-1.92). The duration of use was also examined, but the meta-analysis did not find an effect when subcategorized in durations of $>1$ year, $1-3$ years and more than 3 years. For BRCA2 mutation carriers, a meta-analysis of two case-control studies showed no association with use of OC $(\mathrm{ES}=1.04 ; 95 \%$ CI 0.81-1.32) [25, 28]. The two case-control studies used for the meta-analysis comprised 458 cases and 509 controls with BRCA2 mutation. A meta-analysis of the cohort studies, however, found an association between use of $\mathrm{OC}$ and breast cancer risk in $B R C A 2$ mutation carriers $(\mathrm{ES}=1.85 ; 95 \% \mathrm{CI}$ 1.30-2.64). Two of the case-control studies were used to examine a possible effect of the duration of use [28, 32]. No association was found for a duration of 1-3 years and more than 3 years compared to never use in BRCA2 mutation carriers. There was no information on type and dosage of OC.

In a recent case-only study by Rieder et al. [33], multivariate analysis found an association between prior or current OC use and a younger age at diagnosis in BRCAl/2 mutation carriers (HR $=1.7 ; 95 \%$ CI $1.1-2.05 ; p=0.006)$. The study included $258 B R C A 1$ and $108 B R C A 2$ mutation carriers with a history of breast cancer. The median year of birth in the study population was 1965 . Median age at diagnosis was 58 for women who were born earlier and 42 years for women who were born in or after 1965. The authors paralleled these findings with the fact that in the later birth cohort, the probability of having experienced pregnancies was lower and $\mathrm{OC}$ use more likely. No association was found between breast cancer onset and duration of $\mathrm{OC}(\mathrm{HR}=1.00 ; 95 \%$ CI 0.99-1.00) or starting age (HR=1.03; 95\% CI 0.8-1.3). Differences in type or dosage of OC were not described.

Park et al. [34] recruited $581 B R C A$ mutation carriers for a retrospective cohort study. The study included 222 BRCAI mutation carriers, 168 of which with a history of breast cancer, and 359 BRCA2 mutation carriers, 109 with a history of breast cancer, respectively. Also, the study included non-carriers with positive family history or other high-risk criteria. Use of OC was associated with breast cancer only for noncarriers (HR $=3.99,95 \%$ CI 1.65-9.67), but no association was found for BRCAl ( $\mathrm{HR}=1.24 ; 95 \% \mathrm{CI} 0.45-3.40)$ and $B R C A 2$ mutation carriers (HR $=0.71 ; 95 \%$ CI $0.21-2.37$ ). Type and dosage of $\mathrm{OC}$ as well as duration of use or age at first start were not examined. Study design was retrospective and the study population consisted of Asian BRCA mutation carriers. 
In the most recent study, Schrijver et al. [35] included a total of $6030 B R C A 1$ and $3809 B R C A 2$ mutation carriers to perform prospective, left-truncated retrospective and fullcohort retrospective analyses. Women in the prospective cohort had no history of cancer or risk-reducing mastectomy at the time of inclusion. Follow-up started at birth in the full-cohort retrospective analysis. The left-truncated cohort included only BRCA mutation carriers without a history of cancer or risk-reducing mastectomy at the start of follow-up, 5 years preceding the baseline questionnaire.

For BRCA1 mutation carriers, the prospective analysis found no association between use of $\mathrm{OC}$ and breast cancer risk $(\mathrm{HR}=1.08 ; 95 \%$ CI $0.75-1.5)$. No association was found with the total duration of use, age at first use, recency of use or duration of use before FFTP in the prospective cohort. In contrast, both the left-truncated $(\mathrm{HR}=1.26 ; 95 \% \mathrm{CI} 1.06-1.51)$ and the full-cohort retrospective analysis $(\mathrm{HR}=1.39 ; 95 \% \mathrm{CI} 1.23-1.58)$ found an increase in risk associated with use of OC in BRCAl mutation carriers. There was an inverse correlation between increase in risk and lifetime duration of use ( $p$ trend $=0.01)$ as well as duration of use before FFTP $(p$ trend $=0.001)$ in both retrospective cohorts. When stratified by age, the left-truncated cohort analysis indicated that the trend associated with OC use before FFTP was restricted to women at the age of $\leq 35$ years ( $p$ difference $=0.08$ ). Additionally, an increased risk with younger age at first OC use $(p$ trend $<0.01)$ and longer duration of use after FFTP ( $p$ trend $=0.02$ ) was observed in the fullcohort, but not in the left-truncated cohort. Stratification by age was not possible in the prospective cohort due to small sample size.

For $B R C A 2$ mutation carriers, the prospective analysis showed an increase in risk associated with use of OC (HR $=1.75 ; 95 \%$ CI 1.03-2.9). The results of the retrospective analyses were inconsistent: the left-truncated analysis found no association ( $\mathrm{HR}=1.06$; $95 \% \mathrm{CI} 0.85-1.33$ ), while the full-cohort retrospective analysis showed an increase in breast cancer risk associated with use of $\mathrm{OC}(\mathrm{HR}=1.52$; 95\% CI 1.28-1.81). An increased risk for women with younger age at first use $(p$ trend $<0.01)$ and an association with longer duration of use ( $p$ trend $=0.001$ ), especially for use before FFTP ( $p$ trend $=0.002$ ), were observed in the full-cohort, but not in the left-truncated and the prospective cohort.

Possible explanations for the inconsistencies between the prospective and the retrospective analyses provided by the authors were a survival bias or an underrepresentation of young women in the prospective cohort. In this cohort, the proportion of young women with breast cancer before the age of 35 years was lowest for both $B R C A 1$ and $B R C A 2$ mutation carriers (14.1 and 8.2\%, respectively). Furthermore, the power of the prospective analysis might have been too low to detect trends associated with duration of use or starting age. The authors conclude that the safety of longterm $\mathrm{OC}$ use in BRCA mutation carriers remains uncertain.

\section{Discussion}

For ovarian cancer, current data confirm a risk reduction associated with use of OC in BRCA mutation carriers. Several studies also describe an inverse correlation with the duration of OC use $[5,6,11,13,19]$.

Data on breast cancer risk associated with OC use in $B R C A$ mutation carriers are heterogeneous. Some of the studies report an increase in risk associated with use of OC $[11,13]$, while others did not find an association between breast cancer risk and current preparations of OC $[6,13$, $15,34]$. In some studies, the association was limited to early-onset breast cancer with diagnosis before 40 years of age and/or associated with young age of under 20 years at first start of OC [20, 30, 33]. Some of the studies examined the duration of OC use and showed no significant association with breast cancer risk [6, 20,33]. The review by Cibula which showed a mild to moderate increase in breast cancer risk associated with use of OC showed a further increase in risk when the duration of OC use was at least 4 years before FFTP; the observed HR was comparable with that in women without family history (BRCA1: $\mathrm{HR}=1.49 ; 95 \% \mathrm{CI} 1.05-2.11$. BRCA $2: \mathrm{HR}=2.58 ; 95 \%$ CI 1.21-5.49) [11]. The most recent publication by Schrijver et al. [35] showed similar results. The study found an increase in risk for BRCAl mutation carriers associated with use of OC in both the left-truncated and the fullcohort retrospective analysis and an inverse correlation with duration of use before FFTP which, however, was restricted to women at the age of $\leq 35$ years in the lefttruncated retrospective cohort ( $p$ difference $=0.08$ ). For $B R C A 2$ mutation carriers, a retrospective analysis showed an increase in risk associated with use of OC only in the full-cohort and an association with longer duration of use ( $p$ trend $=0.001)$, especially before FFTP $(p$ trend $=0.002$ ). In most of the studies, however, differences in type or dosage of OC were not described. According to the available data, a reliable statement on possible effects of different formulations can therefore not be made.

Overall, data on the risk of OC use in $B R C A$ mutation carriers are limited. Almost all of the available studies are retrospective and especially for $B R C A 2$ mutation carriers, study populations were often small. Apart from known problems of retrospective studies as recruitment bias and survival bias, the here discussed studies have multiple limitations. Study designs were different and genetic testing for controls not always complete. Partly, women with risk-reducing bilateral oophorectomy or prophylactic 
mastectomy were included $[5,11]$. In some of the studies, specific ethnic subgroups were overrepresented $[5,15]$.

Risk of breast cancer is high in BRCA1/2 mutation carriers, and one or several modifiers with a small effect that is multiplicative can have a significant impact in a high-risk subgroup. This was postulated for low penetrant genetic variants in the past [36]. The only prospective study by Schrijver et al. [35] showed heterogenous results. Being one of the biggest cohort studies to date, the power to stratify for age groups of exposure and for age of onset of breast cancer was still too low. A relevant risk elevating effect of OC use for breast cancer is so far not proven but especially in the specific age group of young $B R C A 1 / 2$ mutation carriers before the age of 40 years is still possible. Larger prospective cohort studies with longer followup and power to stratify for age groups of exposure and for age of onset of breast cancer are therefore urgently needed.

When illuminating the effects and safety of $\mathrm{OC}$ in $B R C A 1 / 2$ mutation carriers, many different aspects must be taken into consideration. The protective effect for ovarian cancer is opposed to the possible increase in breast cancer risk. After having had a risk-reducing mastectomy or bilateral salpingo-oophorectomy (BSO) in the past, separate evaluation of the modifying effect of OC use is helpful for further individual decisions. But giving clinical recommendations are complicated by the fact that potential interaction between external hormonal exposure and other modifying genetic or non-genetic risk factors and its influence on cancer risk are not yet clear.

As mentioned above, data are limited in number as well as study types. Prospective randomised studies are not likely to be conducted due to ethical reasons as well as safety aspects. Therefore, clinical registries will be needed for further evaluation. Additionally, linkage with national registries is necessary to improve the follow-up of the participants. The prospective cohort study of the German Consortium Hereditary Breast and Ovarian Cancer (GC-HBOC) is conducting the HerediCaRe registry, which is part of the International BRCAl and BRCA2 Carrier Cohort Study (IBCCS) [37], one of the largest registries for hereditary breast and ovarian cancer. With the result of several prospective cohort studies, finally a level of evidence (LOE) of 2a according to Oxford nomenclature will be achievable. Until then, carriers of mutations in the genes $B R C A 1$ and $B R C A 2$ as well as in other less frequent cancer genes should be taken care of under trial conditions. This is very important since uptake of risk-reducing BSO is high and even in well-conducted prospective cohort studies it is going to be difficult to accumulate sufficient follow-up time to definitely answer the question of ovarian cancer risk modulation in carriers by OC use.

Oral contraception leads to a risk reduction of ovarian cancer also in BRCA1/2 mutation carriers. An increase in breast cancer risk due to $\mathrm{OC}$ cannot be excluded. Even though ovarian cancer risk is lower with $\mathrm{OC}$ use, it stays elevated and timely risk-reducing BSO is recommended at the age of 40 years for $B R C A 1$ and at the age of 45 years for $B R C A 2$ mutation carriers. Adequate hormonal therapy to avoid postmenopausal symptoms and chronic diseases resulting from low oestrogen levels such as osteoporosis and myocardial infarction is recommended until the age of 50 years. Women with BRCA1/2 mutation who consider OC use have to be informed that this method may lead to an increase in breast cancer risk. OC may be used for contraception in women with $B R C A 1 / 2$ mutation, but they have to be informed about alternative methods. When no contraception is needed, OCs should not be used for the prevention of ovarian cancer in this population to avoid a possible increase in breast cancer risk.

Author contribution DH: Literature search, data analysis, manuscript writing. SS: Data analysis, manuscript writing. KK, GE: Manuscript editing. OO: Project development, manuscript editing.

Funding Open Access funding enabled and organized by Projekt DEAL

\section{Compliance with ethical standards}

Conflict of interest The authors declare that they have no conflict of interest.

Open Access This article is licensed under a Creative Commons Attribution 4.0 International License, which permits use, sharing, adaptation, distribution and reproduction in any medium or format, as long as you give appropriate credit to the original author(s) and the source, provide a link to the Creative Commons licence, and indicate if changes were made. The images or other third party material in this article are included in the article's Creative Commons licence, unless indicated otherwise in a credit line to the material. If material is not included in the article's Creative Commons licence and your intended use is not permitted by statutory regulation or exceeds the permitted use, you will need to obtain permission directly from the copyright holder. To view a copy of this licence, visit http://creativecommons.org/licenses/by/4.0/.

\section{References}

1. Kuchenbaecker KB, Hopper JL, Barnes DR, Phillips K-A, Mooij TM, Roos-Blom M-J et al (2017) Risks of breast, ovarian, and contralateral breast cancer for BRCA1 and BRCA 2 mutation carriers. JAMA 317(23):2402-2416

2. Paluch-Shimon S, Cardoso F, Sessa C, Balmana J, Cardoso MJ, Gilbert $\mathrm{F}$ et al (2016) Prevention and screening in BRCA mutation carriers and other breast/ovarian hereditary cancer syndromes: ESMO Clinical Practice Guidelines for cancer prevention and screening. Ann Oncol 27(suppl 5):v103-v110

3. Mørch LS, Hannaford PC, Lidegaard Ø (2018) Contemporary hormonal contraception and the risk of breast cancer. N Engl J Med 378(13):1265-1266

4. Beral V, Doll R, Hermon C, Peto R, Reeves G (2008) Ovarian cancer and oral contraceptives: collaborative reanalysis of data 
from 45 epidemiological studies including 23,257 women with ovarian cancer and 87,303 controls. Lancet 371(9609):303-314

5. Narod SA, Risch H, Moslehi R, Dørum A, Neuhausen S, Olsson H et al (1998) Oral contraceptives and the risk of hereditary ovarian cancer. Hereditary Ovarian Cancer Clinical Study Group. N Engl J Med 339(7):424-428

6. Iodice S, Barile M, Rotmensz N, Feroce I, Bonanni B, Radice $P$ et al (2010) Oral contraceptive use and breast or ovarian cancer risk in BRCA1/2 carriers: a meta-analysis. Eur $\mathrm{J}$ Cancer 46(12):2275-2284

7. Whittemore AS, Balise RR, Pharoah PDP, Dicioccio RA, Oakley-Girvan I, Ramus SJ et al (2004) Oral contraceptive use and ovarian cancer risk among carriers of BRCA1 or BRCA2 mutations. Br J Cancer 91(11):1911-1915

8. Antoniou AC, Rookus M, Andrieu N, Brohet R, Chang-Claude J, Peock S et al (2009) Reproductive and hormonal factors, and ovarian cancer risk for BRCA1 and BRCA2 mutation carriers: results from the International BRCA1/2 Carrier Cohort Study. Cancer Epidemiol Biomarkers Prev 18(2):601-610

9. Runnebaum IB, Wang-Gohrke S, Vesprini D, Kreienberg R, Lynch H, Moslehi R et al (2001) Progesterone receptor variant increases ovarian cancer risk in BRCA1 and BRCA2 mutation carriers who were never exposed to oral contraceptives. Pharmacogenetics 11(7):635-638

10. McLaughlin JR, Risch HA, Lubinski J, Moller P, Ghadirian P, Lynch H et al (2007) Reproductive risk factors for ovarian cancer in carriers of BRCA1 or BRCA2 mutations: a case-control study. Lancet Oncol 8(1):26-34

11. Cibula D, Gompel A, Mueck AO, La Vecchia C, Hannaford PC, Skouby SO et al (2010) Hormonal contraception and risk of cancer. Hum Reprod Update 16(6):631-650

12. Modan B, Hartge P, Hirsh-Yechezkel G, Chetrit A, Lubin F, Beller U et al (2001) Parity, oral contraceptives, and the risk of ovarian cancer among carriers and noncarriers of a BRCA1 or BRCA2 mutation. N Engl J Med 345(4):235-240

13. Cibula D, Zikan M, Dusek L, Majek O (2011) Oral contraceptives and risk of ovarian and breast cancers in BRCA mutation carriers: a meta-analysis. Expert Rev Anticancer Ther 11(8):1197-1207

14. Gronwald J, Byrski T, Huzarski T, Cybulski C, Sun P, Tulman A et al (2006) Influence of selected lifestyle factors on breast and ovarian cancer risk in BRCA1 mutation carriers from Poland. Breast Cancer Res Treat 95(2):105-109

15. Moorman PG, Havrilesky LJ, Gierisch JM, Coeytaux RR, Lowery WJ, Peragallo Urrutia R et al (2013) Oral contraceptives and risk of ovarian cancer and breast cancer among high-risk women: a systematic review and meta-analysis. J Clin Oncol 31(33):4188-4198

16. Friebel TM, Domchek SM, Rebbeck TR (2014) Modifiers of cancer risk in BRCA1 and BRCA2 mutation carriers: systematic review and meta-analysis. J Natl Cancer Inst 106(6):dju091

17. Narod SA, Sun P, Ghadirian P, Lynch H, Isaacs C, Garber J et al (2001) Tubal ligation and risk of ovarian cancer in carriers of BRCA1 or BRCA2 mutations: a case-control study. Lancet 357(9267): 1467-1470

18. Vicus D, Rosen B, Lubinski J, Domchek S, Kauff ND, Lynch HT et al (2009) Tamoxifen and the risk of ovarian cancer in BRCA1 mutation carriers. Gynecol Oncol 115(1):135-137

19. Perri T, Lifshitz D, Sadetzki S, Oberman B, Meirow D, BenBaruch G et al (2015) Fertility treatments and invasive epithelial ovarian cancer risk in Jewish Israeli BRCA1 or BRCA2 mutation carriers. Fertil Steril 103(5):1305-1312

20. Pasanisi P, Hédelin G, Berrino J, Chang-Claude J, Hermann S, Steel M et al (2009) Oral contraceptive use and BRCA penetrance: a case-only study. Cancer Epidemiol Biomarkers Prev 18(7):2107-2113
21. Heimdal K, Skovlund E, Møller P (2002) Oral contraceptives and risk of familial breast cancer. Cancer Detect Prev 26(1):23-27

22. Jernström H, Loman N, Johannsson OT, Borg A, Olsson H (2005) Impact of teenage oral contraceptive use in a population-based series of early-onset breast cancer cases who have undergone BRCA mutation testing. Eur J Cancer 41(15):2312-2320

23. Sade RBB, Chetrit A, Figer A, Papa MZ, Flex D, Rizel S et al (2006) Hormone replacement therapy is more prevalent among Jewish BRCA1/2 mutation carriers. Eur J Cancer 42(5):650-655

24. Ursin G, Henderson BE, Haile RW, Pike MC, Zhou N, Diep A et al (1997) Does oral contraceptive use increase the risk of breast cancer in women with BRCA1/BRCA2 mutations more than in other women? Cancer Res 57(17):3678-3681

25. Narod SA, Dubé M-P, Klijn J, Lubinski J, Lynch HT, Ghadirian $P$ et al (2002) Oral contraceptives and the risk of breast cancer in BRCA1 and BRCA2 mutation carriers. J Natl Cancer Inst 94(23): 1773-1779

26. Brohet RM, Goldgar DE, Easton DF, Antoniou AC, Andrieu $\mathrm{N}$, Chang-Claude J et al (2007) Oral contraceptives and breast cancer risk in the international BRCA1/2 carrier cohort study: a report from EMBRACE, GENEPSO, GEO-HEBON, and the IBCCS Collaborating Group. J Clin Oncol 25(25):3831-3836

27. Milne RL, Knight JA, John EM, Dite GS, Balbuena R, Ziogas A et al (2005) Oral contraceptive use and risk of early-onset breast cancer in carriers and noncarriers of BRCA1 and BRCA2 mutations. Cancer Epidemiol Biomarkers Prev 14(2):350-356

28. Haile RW, Thomas DC, McGuire V, Felberg A, John EM, Milne RL et al (2006) BRCA1 and BRCA2 mutation carriers, oral contraceptive use, and breast cancer before age 50. Cancer Epidemiol Biomarkers Prev 15(10):1863-1870

29. Bernholtz S, Laitman Y, Kaufman B, Paluch Shimon S, Friedman E (2011) Cancer risk in Jewish BRCA1 and BRCA2 mutation carriers: effects of oral contraceptive use and parental origin of mutation. Breast Cancer Res Treat 129(2):557-563

30. Kotsopoulos J, Lubinski J, Moller P, Lynch HT, Singer CF, Eng $\mathrm{C}$ et al (2014) Timing of oral contraceptive use and the risk of breast cancer in BRCA1 mutation carriers. Breast Cancer Res Treat 143(3):579-586

31. Gronwald J, Glass K, Rosen B, Karlan B, Tung N, Neuhausen SL et al (2016) Treatment of infertility does not increase the risk of ovarian cancer among women with a BRCA1 or BRCA2 mutation. Fertil Steril 105(3):781-785

32. Kotsopoulos J, Lubinski J, Lynch HT, Kim-Sing C, Neuhausen S, Demsky R et al (2012) Oophorectomy after menopause and the risk of breast cancer in BRCA1 and BRCA2 mutation carriers. Cancer Epidemiol Biomarkers Prev 21(7):1089-1096

33. Rieder V, Salama M, Glöckner L, Muhr D, Berger A, Tea M-K et al (2016) Effect of lifestyle and reproductive factors on the onset of breast cancer in female BRCA 1 and 2 mutation carriers. Mol Genet Genomic Med 4(2):172-177

34. Park B, Hopper JL, Win AK, Dowty JG, Sung HK, Ahn C et al (2017) Reproductive factors as risk modifiers of breast cancer in BRCA mutation carriers and high-risk non-carriers. Oncotarget 8(60):102110-102118

35. Schrijver LH, Olsson H, Phillips K-A, Terry MB, Goldgar DE, Kast $\mathrm{K}$ et al (2018) Oral contraceptive use and breast cancer risk: retrospective and prospective analyses from a BRCA1 and BRCA2 mutation carrier cohort study. JNCI Cancer Spectr 2(2):pky023

36. Antoniou AC, Spurdle AB, Sinilnikova OM, Healey S, Pooley KA, Schmutzler RK et al (2008) Common breast cancer-predisposition alleles are associated with breast cancer risk in BRCA1 and BRCA2 mutation carriers. Am J Hum Genet 82(4):937-948

37. Antoniou AC, Goldgar DE, Andrieu N, Chang-Claude J, Brohet $\mathrm{R}$, Rookus MA et al (2005) A weighted cohort approach for 
analysing factors modifying disease risks in carriers of high-risk susceptibility genes. Genet Epidemiol 29(1):1-11

38. McGuire V, Felberg A, Mills M, Ostrow KL, DiCioccio R, John EM et al (2004) Relation of contraceptive and reproductive history to ovarian cancer risk in carriers and noncarriers of BRCA1 gene mutations. Am J Epidemiol 160(7):613-618

39. Jernström H, Lerman C, Ghadirian P, Lynch HT, Weber B, Garber J et al (1999) Pregnancy and risk of early breast cancer in carriers of BRCA1 and BRCA2. Lancet 354(9193):1846-1850

40. Lee E, Ma H, McKean-Cowdin R, van den Berg D, Bernstein L, Henderson BE et al (2008) Effect of reproductive factors and oral contraceptives on breast cancer risk in BRCA1/2 mutation carriers and noncarriers: results from a population-based study. Cancer Epidemiol Biomarkers Prev 17(11):3170-3178
41. Figueiredo JC, Haile RW, Bernstein L, Malone KE, Largent J, Langholz B et al (2010) Oral contraceptives and postmenopausal hormones and risk of contralateral breast cancer among BRCA1 and BRCA2 mutation carriers and noncarriers: the WECARE Study. Breast Cancer Res Treat 120(1):175-183

42. Atchley DP, Albarracin CT, Lopez A, Valero V, Amos CI, Gonzalez-Angulo AM et al (2008) Clinical and pathologic characteristics of patients with BRCA-positive and BRCA-negative breast cancer. J Clin Oncol 26(26):4282-4288

Publisher's Note Springer Nature remains neutral with regard to jurisdictional claims in published maps and institutional affiliations. 